\title{
Die Schweiz braucht ein starkes Tabakproduktegesetz
}

\author{
Jacques Cornuz \\ Prof. Dr. med., Direktor der Medizinischen Poliklinik des Universitätsspitals Lausanne
}

\begin{abstract}
2015 steht der Welttag ohne Tabak vom 31. Mai unter dem Thema illegaler Handel. Gemäss der Weltgesundheitsorganisation wird jede zehnte Zigarette illegal verkauft. Das neue Tabakproduktegesetz bietet die Chance, auf gesetzlicher Ebene die Tabakprävention zu stärken. Die gesundheitlichen Folgeschäden von Nikotin rechtfertigen, für eine gesündere Schweiz dem Handel mit Tabakprodukten strenge Regeln aufzuerlegen.
\end{abstract}

Im letzten Bericht des US-Surgeon General, fünfzig Jahre nach dem ersten von 1964, verlängerte sich die Liste der tabakbedingten Erkrankungen um weitere häufige Krankheiten wie Diabetes mellitus Typ 2, Rheumatoide Arthritis und Hepatozelluläres Karzinom. Bekanntlich stirbt die Hälfte der Konsumentinnen und Konsumenten an tabakbedingten Krankheiten: ein «Russisches Roulette» mit drei statt nur einer Kugel. Die tödlichen Auswirkungen des Rauchens rechtfertigen, dass der Gesetzgeber dem Handel mit Tabakprodukten enge Grenzen setzt.

Der Vorentwurf des Bundesrates zum neuen Tabakproduktegesetz weist aber aus Sicht einer kohärenten Tabakprävention verschiedene Lücken auf. Zusätzliche Massnahmen sind notwendig. Denn rechtliche Rahmenbedingungen können den Tabakkonsum nur dann entscheidend beeinflussen, wenn sie die Vermarktung von Tabakwaren limitieren. Die Gesundheit der Bevölkerung hat Vorrang vor den wirtschaftlichen Interessen der Tabakindustrie.

\section{Werbung und Verkauf}

Die Wirkung der Tabakwerbung besonders auf Jugendliche ist heute unbestritten. Doch gerade bei der Ver-

Fax 0213146099

www.pmu-lausanne.ch marktung von Tabakwaren sind die vom Bundesrat vorgeschlagenen Massnahmen ungenügend. Das neue Gesetz sollte besonders die Werbung am Verkaufsort und die Verkaufsförderung durch Rabatte untersagen.

\section{E-Zigaretten}

Der bundesrätliche Vorentwurf erlaubt neu den Verkauf von E-Zigaretten mit Nikotin. Dies stimmt überein mit einem kürzlich publizierten Bericht von Schweizer Experten [1]. Aber der Zugang muss ebenso begrenzt sein wie bei der Tabakzigarette. Deshalb sollen E-Zigaretten mit und ohne Nikotin den gleichen Bestimmungen wie die anderen Tabakprodukte unterliegen, auch hinsichtlich Werbung, Promotion und Sponsoring.

Auf der Tobacco Control Scale 2013 in Europe, veröffentlicht von der Association of European Cancer Leagues, erreicht die Schweiz nur 45 von 100 Punkten. Vor allem fehlt eine umfassende Begrenzung der Vermarktung. Das neue Tabakproduktegesetz ist für die Schweiz eine einmalige Gelegenheit, den Rückstand in der gesetzlichen Regelung von Tabakprodukten aufzuholen.

\section{Informationen}

- Auf der Webseite der Arbeitsgemeinschaft Tabakprävention finden Sie eine Rubrik zum Tabakproduktegesetz unter www.at-schweiz.ch $\rightarrow$ Fakten $\rightarrow$ Tabakproduktegesetz.

- Alle Formen von Werbung, Verkaufsförderung und Sponsoring für Tabakwaren in der Schweiz dokumentiert die Webseite www.beobachtung-marke ting-tabak.ch

1 Blaser J, Cornuz J. Experts' consensus on use of electronic cigarettes: a Delphi survey from Switzerland. BMJ Open. 2015; Apr 15;5(4). 